Rakenteiden Mekaniikka (Journal of Structural Mechanics)

Vol. 52, No 3, 2019, pp. 192-199

http://rakenteidenmekaniikka.journal.fi/index

https://doi.org/10.23998/rm.75555

(C) 2019 The Authors

Open access under license CC BY 4.0

\title{
A modified four-node rectangular element
}

\author{
Jouni Freund ${ }^{1}$ and Eero-Matti Salonen
}

Summary. The sensitized principle of virtual work is applied to modify the stiffness matrix of the ordinary four-node rectangular element by sensitizing terms. The sensitizing parameter values are determined by the single-element strain energy test. The reference solutions used are of bending mode types and their application removes the so-called parasitic shear behavior. A stiffness matrix of good quality is obtained corresponding exactly to an earlier formulation using incompatible modes.

Key words: structural mechanics, sensitized principle of virtual work, four-node rectangular element

Received 1 October 2018. Accepted 12 December 2018. Published online 12 September 2019.

\section{Introduction}

The four-node rectangular element sketched in Figure 1 is one of the first elements applied in structural mechanics, e.g. [1].

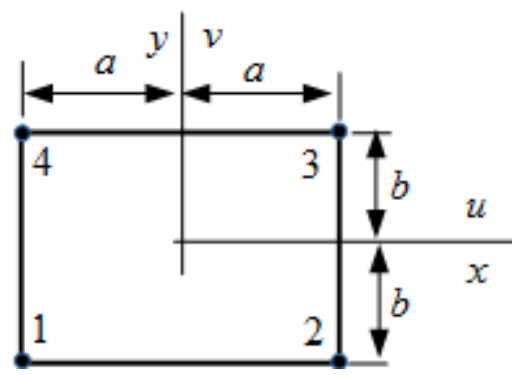

Figure 1. Eight degree of freedom linear (also called bilinear) element.

The assumed displacement components in the $x$ - and $y$-directions are of the forms

$$
\begin{aligned}
& u=\alpha_{1}+\alpha_{2} x+\alpha_{3} y+\alpha_{4} x y, \\
& v=\beta_{1}+\beta_{2} x+\beta_{3} y+\beta_{4} x y .
\end{aligned}
$$

${ }^{1}$ Corresponding author: jouni.freund@aalto.fi 
Standard manipulations give then the matrix type representation

$$
\left\{\begin{array}{l}
u \\
v
\end{array}\right\}=\left[\begin{array}{cccccccc}
N_{1} & 0 & N_{2} & 0 & N_{3} & 0 & N_{4} & 0 \\
0 & N_{1} & 0 & N_{2} & 0 & N_{3} & 0 & N_{4}
\end{array}\right]\{\mathbf{a}\}
$$

with the nodal displacement column matrix

$$
\{\mathbf{a}\}=\left[\begin{array}{llllllll}
u_{1} & v_{1} & u_{2} & v_{2} & u_{3} & v_{3} & u_{4} & v_{4}
\end{array}\right]^{\mathrm{T}}
$$

and with the shape functions

$$
\begin{aligned}
& N_{1}=\frac{1}{4 a b}(a-x)(b-y), \quad N_{2}=\frac{1}{4 a b}(a+x)(b-y), \\
& N_{3}=\frac{1}{4 a b}(a+x)(b+y), \quad N_{4}=\frac{1}{4 a b}(a-x)(b+y) .
\end{aligned}
$$

Details concerning the application of this element in plane stress or plane strain elastic cases are reported e.g. in [2] and [3]. Reference [2] explains also in a very illustrative way certain deficiencies (for example the so-called parasitic shear) of the element and ways to improve its behavior. A more detailed discussion about the effect of domain slenderness, different locking phenomena, and properties of some formulations aiming at improved performance is available in [3]. In the following, we present still another attempt to improve the element behavior based now on the sensitized principle of virtual work.

\section{Sensitized finite element method}

The sensitized principle of virtual work is described in reference [4] and in fact especially in the two-dimensional continuum case so the formulas presented are directly applicable here. We therefore just record the necessary expressions.

We define

$$
\{\mathbf{u}\}=\left\{\begin{array}{l}
u \\
v
\end{array}\right\}, \quad\{\mathbf{b}\}=\left\{\begin{array}{l}
b_{x} \\
b_{y}
\end{array}\right\}, \quad\{\mathbf{t}\}=\left\{\begin{array}{l}
t_{x} \\
t_{y}
\end{array}\right\}, \quad\{\boldsymbol{\sigma}\}=\left\{\begin{array}{c}
\sigma_{x} \\
\sigma_{y} \\
\tau_{x y}
\end{array}\right\}, \quad\{\boldsymbol{\varepsilon}\}=\left\{\begin{array}{c}
\varepsilon_{x} \\
\varepsilon_{y} \\
\gamma_{x y}
\end{array}\right\}
$$

and further

$$
\{\boldsymbol{\varepsilon}\}=[\mathbf{S}]\{\mathbf{u}\},
$$

where

$$
[\mathbf{S}]=\left[\begin{array}{cc}
\partial / \partial x & 0 \\
0 & \partial / \partial y \\
\partial / \partial y & \partial / \partial x
\end{array}\right]
$$


The local equilibrium equations can be written as

$$
[\mathbf{E}]\{\boldsymbol{\sigma}\}+\{\mathbf{b}\}=\{\boldsymbol{0}\}
$$

with

$$
[\mathbf{E}]=[\mathbf{S}]^{\mathrm{T}}=\left[\begin{array}{ccc}
\partial / \partial x & 0 & \partial / \partial y \\
0 & \partial / \partial y & \partial / \partial x
\end{array}\right]
$$

The sensitizing parameter matrix

$$
[\boldsymbol{\tau}]=\left[\begin{array}{ll}
\tau_{11} & \tau_{12} \\
\tau_{21} & \tau_{22}
\end{array}\right]
$$

can be taken symmetric $\left(\tau_{21}=\tau_{12}\right)$. The finite element displacement approximation is written as

$$
\{\mathbf{u}\}=[\mathbf{N}]\{\mathbf{a}\} .
$$

Differing from (3), here we consider temporarily the whole structure and thus the column matrix $\{\mathbf{a}\}$ is considered to contain all the nodal displacements of the structure and $[\mathbf{N}]$ is thus the corresponding global shape function matrix. We assume elastic material with the constitutive relation

$$
\{\boldsymbol{\sigma}\}=[\mathbf{D}]\{\boldsymbol{\varepsilon}\} .
$$

We introduce the shorthand notations

$$
[\mathbf{B}] \equiv[\mathbf{S}][\mathbf{N}]
$$

and

$$
[\mathbf{C}] \equiv[\mathbf{E}][\mathbf{D}][\mathbf{B}]
$$

The following finite element equations are arrived at

$$
[\mathbf{K}]\{\mathbf{a}\}=\{\mathbf{f}\},
$$

where

$$
[\mathbf{K}] \equiv\left[\mathbf{K}_{\mathrm{O}}\right]+\left[\mathbf{K}_{\mathrm{S}}\right] \equiv \int_{A}[\mathbf{B}]^{\mathrm{T}}[\mathbf{D}][\mathbf{B}] \mathrm{d} A+\int_{A}[\mathbf{C}]^{\mathrm{T}}[\boldsymbol{\tau}][\mathbf{C}] \mathrm{d} A
$$

and

$$
\{\mathbf{f}\} \equiv\left\{\mathbf{f}_{\mathrm{O}}\right\}+\left\{\mathbf{f}_{\mathrm{S}}\right\} \equiv \int_{A}[\mathbf{N}]^{\mathrm{T}}\{\mathbf{b}\} \mathrm{d} A+\int_{S}[\mathbf{N}]^{\mathrm{T}}\{\mathbf{t}\} \mathrm{d} s-\int_{A}[\mathbf{C}]^{\mathrm{T}}[\boldsymbol{\tau}]\{\mathbf{b}\} \mathrm{d} A .
$$


The subscripts $\mathrm{O}$ and $\mathrm{S}$ refer to the terms emerging from the ordinary and sensitizing parts of the formulas, respectively.

\section{The rectangular element}

We now return to the element discussed in the "Introduction" section. The finite element formulas given in the preceding section can be used also for an element by just taking into account the correct sizes of the corresponding matrices. However, we then cannot speak any more about element equations but of the element contributions

$$
\{\mathbf{F}\} \equiv[\mathbf{K}]\{\mathbf{a}\}-\{\mathbf{f}\}
$$

to the global discrete equations. For instance, the column matrix $\{\mathbf{a}\}$ in (19) is now the one in (4).

We consider here the plane stress case and isotropic material so in matrix $[\mathbf{D}]$ in formula (13)

$$
\begin{gathered}
D_{11}=D_{22}=\frac{E}{1-v^{2}}, \quad D_{33}=\frac{E}{2(1+v)}, \quad D_{21}=D_{12}=\frac{E v}{1-v^{2}}, \\
D_{31}=D_{13}=0, \quad D_{32}=D_{23}=0,
\end{gathered}
$$

where $E$ is Young's modulus and $v$ Poisson's ratio considered as constants in an element.

Straightforward calculations give

$$
[\mathbf{B}]=\frac{1}{4 a b}\left[\begin{array}{cccccccc}
-b+y & 0 & b-y & 0 & b+y & 0 & -b-y & 0 \\
0 & -a+x & 0 & -a-x & 0 & a+x & 0 & a-x \\
-a+x & -b+y & -a-x & b-y & a+x & b+y & a-x & -b-y
\end{array}\right]
$$

and

$$
[\mathbf{C}]=\frac{E}{8 a b(1-v)}\left[\begin{array}{cccccccc}
0 & 1 & 0 & -1 & 0 & 1 & 0 & -1 \\
1 & 0 & -1 & 0 & 1 & 0 & -1 & 0
\end{array}\right] \text {. }
$$

It is seen that the terms in the $[\mathbf{C}]$-matrix are here constants with respect to position. Matrices $\left[\mathbf{K}_{\mathrm{O}}\right]$ and $\left[\mathbf{K}_{\mathrm{S}}\right]$ for the element are conveniently evaluated say by the Mathematica program [5]. For the former matrix we record here just as samples four typical terms:

$$
\begin{aligned}
& \left(K_{\mathrm{O}}\right)_{11}=\frac{E}{12\left(1-v^{2}\right)}\left[4 \frac{b}{a}+2(1-v) \frac{a}{b}\right], \quad\left(K_{\mathrm{O}}\right)_{12}=\frac{E}{12\left(1-v^{2}\right)} \frac{3}{2}(1+v), \\
& \left(K_{\mathrm{O}}\right)_{13}=\frac{E}{12\left(1-v^{2}\right)}\left[-4 \frac{b}{a}+(1-v) \frac{a}{b}\right], \quad\left(K_{\mathrm{O}}\right)_{14}=\frac{E}{12\left(1-v^{2}\right)}\left(-\frac{3}{2}+\frac{9}{2} v\right) .
\end{aligned}
$$

The latter matrix obtains a simple form 


$$
\left[\mathbf{K}_{\mathrm{S}}\right]=\frac{E^{2}}{16 a b(1-v)^{2}}\left[\begin{array}{cccccccc}
\tau_{22} & \tau_{21} & -\tau_{22} & -\tau_{21} & \tau_{22} & \tau_{21} & -\tau_{22} & -\tau_{21} \\
\tau_{12} & \tau_{11} & -\tau_{12} & -\tau_{11} & \tau_{12} & \tau_{11} & -\tau_{12} & -\tau_{11} \\
-\tau_{22} & -\tau_{21} & \tau_{22} & \tau_{21} & -\tau_{22} & -\tau_{21} & \tau_{22} & \tau_{21} \\
-\tau_{12} & -\tau_{11} & \tau_{12} & \tau_{11} & -\tau_{12} & -\tau_{11} & \tau_{12} & \tau_{11} \\
\tau_{22} & \tau_{21} & -\tau_{22} & -\tau_{21} & \tau_{22} & \tau_{21} & -\tau_{22} & -\tau_{21} \\
\tau_{12} & \tau_{11} & -\tau_{12} & -\tau_{11} & \tau_{12} & \tau_{11} & -\tau_{12} & -\tau_{11} \\
-\tau_{22} & -\tau_{21} & \tau_{22} & \tau_{21} & -\tau_{22} & -\tau_{21} & \tau_{22} & \tau_{21} \\
-\tau_{12} & -\tau_{11} & \tau_{12} & \tau_{11} & -\tau_{12} & -\tau_{11} & \tau_{12} & \tau_{11}
\end{array}\right]
$$

This stiffness matrix has a rather peculiar structure. A short study shows that if the nodal displacements are evaluated from linear displacement expressions like $u=\alpha+\beta x+\gamma y$ or $v=\alpha+\beta x+\gamma y$ (with arbitrary values for the constants $\alpha, \beta$ and $\gamma)$, the resulting nodal forces vanish. The ordinary element with stiffness matrix $\left[\mathbf{K}_{\mathrm{O}}\right]$ is known to pass the patch test (convergence checking test A or B in reference [6]). When the contributions from the sensitized stiffness matrix $\left[\mathbf{K}_{\mathrm{S}}\right]$ are included in the patch test, the nodal equilibrium equations do not get any non-zero additions. Thus, the patch test will be passed for arbitrary values of the sensitizing parameters $\tau_{11}, \tau_{22}$ and $\tau_{21}=\tau_{12}$. This is an important result which obviously gives much freedom for the selection of the parameter values.

\section{Strain energy test}

The idea behind the strain energy test is also explained in [4]. The strain energy of the element evaluated analytically from a reference solution is given by

$$
U_{\mathrm{A}}=\frac{1}{2} \int_{-b}^{b} \int_{-a}^{a}\{\boldsymbol{\varepsilon}\}^{\mathrm{T}}[\mathbf{D}]\{\boldsymbol{\varepsilon}\} \mathrm{d} x \mathrm{~d} y .
$$

The strain energy evaluated from the corresponding element nodal displacement values is

$$
U_{\mathrm{N}}=\frac{1}{2}\{\mathbf{a}\}^{\mathrm{T}}[\mathbf{K}]\{\mathbf{a}\}=\frac{1}{2}\{\mathbf{a}\}^{\mathrm{T}}\left[\mathbf{K}_{\mathrm{O}}\right]\{\mathbf{a}\}+\frac{1}{2}\{\mathbf{a}\}^{\mathrm{T}}\left[\mathbf{K}_{\mathrm{S}}\right]\{\mathbf{a}\}
$$

The test is

$$
U_{\mathrm{N}}=U_{\mathrm{A}} \cdot
$$

This gives an equation containing the sensitizing parameters.

Following the presentation in [2], we consider first specifically the "bending mode"

$$
u=\frac{x y}{a b} \bar{u}, \quad v=0,
$$


where $\bar{u}$ is the magnitude of the horizontal displacement at the element nodes. The corresponding deformation shape is shown in Figure 2 (a).

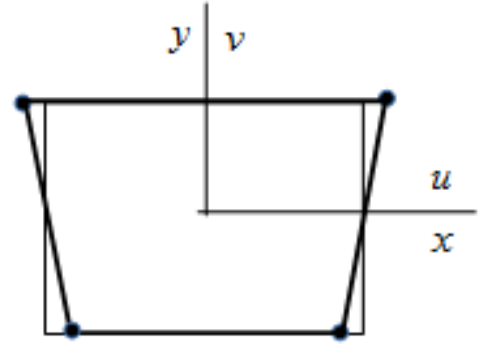

(a)

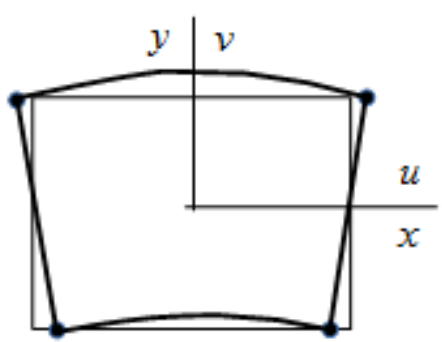

(b)

Figure 2 (a). Deformed element shape due to nodal displacements $\bar{u}$. (b) The corresponding beam segment deformation in pure bending.

However, as explained in [2], we would like to have an actual beam bending mode as in Figure 2 (b) with the solution

$$
u=\frac{x y}{a b} \bar{u}, \quad v=\frac{a}{2 b}\left(1-\frac{x^{2}}{a^{2}}\right) \bar{u}+v \frac{b}{2 a}\left(1-\frac{y^{2}}{b^{2}}\right) \bar{u} .
$$

Thus, our conclusion here is simply that we take (29) as one reference solution.

As again explained in [2], we can consider additionally the vertical "bending mode"

$$
u=0, \quad v=\frac{x y}{a b} \bar{v}
$$

with $\bar{v}$ as the nodal displacements in the vertical direction. Now the corresponding beam segment pure bending solution becomes

$$
u=\frac{b}{2 a}\left(1-\frac{y^{2}}{b^{2}}\right) \bar{v}+v \frac{a}{2 b}\left(1-\frac{x^{2}}{a^{2}}\right) \bar{v}, \quad v=\frac{x y}{a b} \bar{v} .
$$

This is taken as a second reference solution.

In the first case equation (27) becomes

$$
\frac{E\left[2 b^{2}+a^{2}(1-v)\right] \bar{u}^{2}}{3 a b\left(1-v^{2}\right)}+\frac{E^{2} \tau_{22} \bar{u}^{2}}{2 a b(1-v)^{2}}=\frac{2 E b \bar{u}^{2}}{3 a}
$$

and in the second case

$$
\frac{E\left[2 a^{2}+b^{2}(1-v)\right] \bar{v}^{2}}{3 a b\left(1-v^{2}\right)}+\frac{E^{2} \tau_{11} \bar{v}^{2}}{2 a b(1-v)^{2}}=\frac{2 E b \bar{v}^{2}}{3 b} .
$$

The solutions are correspondingly 


$$
\begin{gathered}
\tau_{22}=-\frac{2(1-v)\left[a^{2}(1-v)+2 b^{2} v^{2}\right]}{3 E(1+v)}, \\
\tau_{11}=-\frac{2(1-v)\left[b^{2}(1-v)+2 a^{2} v^{2}\right]}{3 E(1+v)} .
\end{gathered}
$$

Additional reference solution (or solutions) would be needed to determine the parameter $\tau_{21}=\tau_{12}$. In this study we have just simply used the selection (as explained above, from the point of the convergence patch test we are allowed to take any value)

$$
\tau_{21}=\tau_{12}=0 .
$$

We now substitute the values (34), (35) and (36) into (24) to obtain the final sensitizing part of the stiffness matrix. For instance, we obtain

$$
\left(K_{\mathrm{S}}\right)_{11}=-\frac{E}{12\left(1-v^{2}\right)}\left[v^{2} \frac{b}{a}+\frac{(1-v)}{2} \frac{a}{b}\right], \quad\left(K_{\mathrm{S}}\right)_{22}=-\frac{E}{12\left(1-v^{2}\right)}\left[v^{2} \frac{a}{b}+\frac{(1-v)}{2} \frac{b}{a}\right]
$$

The representative terms in (23) are changed by sensitizing to

$$
\begin{gathered}
K_{11}=\frac{E}{12\left(1-v^{2}\right)}\left[\left(4-v^{2}\right) \frac{b}{a}+\frac{3}{2}(1-v) \frac{a}{b}\right], \quad K_{12}=\frac{E}{12\left(1-v^{2}\right)} \frac{3}{2}(1+v), \\
K_{13}=\frac{E}{12\left(1-v^{2}\right)}\left[-\left(4-v^{2}\right) \frac{b}{a}+\frac{3}{2}(1-v) \frac{a}{b}\right], \quad K_{14}=\frac{E}{12\left(1-v^{2}\right)}\left(-\frac{3}{2}+\frac{9}{2} v\right) .
\end{gathered}
$$

The terms $K_{12}$ and $K_{14}$ are seen to remain unchanged.

An incompatible four-node element, called QM6 element, is described in [2] and in more detail in [7] and [8]. It is obtained by adding bending modes of the type $\left[1-(x / a)^{2}\right] a_{1}$ and $\left[1-(y / b)^{2}\right] a_{2}$ to the displacement component representations. This element gives very accurate results. It is rather astonishing that the condensed stiffness matrix terms for the rectangular shape of QM6 recorded in [2, p. 194], coincide exactly with (38) in spite of the different starting points. Based on this, the sensitized modified linear rectangular element should work well in practice and some efforts to extend it to the general quadrilateral shape may be justified.

Concerning the theoretical background, we may quote the following [9, p. 491]: "Incompatible modes always pose a pedagogical dilemma, since a teacher first stresses the importance of compatible fields in developing the single-field elements and then has to dismantle this pedagogical structure by introducing incompatible modes."

\section{Concluding remarks}

Use of the sensitized principle of virtual work can increase the applicability of certain finite elements. This was shown in [4] for the two-node Timoshenko beam element. Similarly, the contents of the present article show that the properties of the standard 
four-node quadrilateral element can be modified beneficially by sensitizing. However, the present study is restricted especially by the use of the selection $\tau_{21}=\tau_{12}=0$. Additional efforts are needed to fully clarify of the possibilities available.

We may finally note the following. In the Timoshenko beam problem treated in reference [4], the sensitized stiffness matrix $\left[\mathbf{K}_{\mathrm{S}}\right]$ is of such a form that it vanishes compared to the ordinary stiffness matrix $\left[\mathbf{K}_{\mathrm{O}}\right]$ with vanishing mesh size. This fact justifies the apparent mathematical crimes performed with respect to continuity of certain terms at element boundaries when just $C^{0}$ continuous approximations are employed. For the present four-node element, matrix $\left[\mathbf{K}_{\mathrm{S}}\right]$ does not vanish with vanishing mesh size. The justification on the use of this element comes now from its behavior in the convergence patch test described above in the text.

\section{References}

[1] O. C. Zienkiewicz and Y.K. Cheung, The Finite Element Method in Structural and Continuum Mechanics, McGraw-Hill, 1967.

[2] D. Cook, Concepts and Applications of Finite Element Analysis, Second Edition, Wiley, 1981.

[3] J. Pitkäranta, The first locking-free plane-elastic finite element: historia mathematica, Computer Methods in Applied Mechanics and Engineering, Volume 190, Issues 11-12, 2000, pp. 1323-1366. https://doi.org/10.1016/S00457825(00)00163-8

[4] J. Freund and E-M. Salonen, Sensitized principle of virtual work and the singleelement strain energy test. Journal of Structural Mechanics, Vol. 49, No.1, 2016, pp.1-13.

[5] Mathematica. https://www.wolfram.com/mathematica/.

[6] O. C. Zienkiewicz and R.L. Taylor, The Finite Element Method, Volume 1: The Basis, Fifth Edition, Butterwort Heinemann, 2000.

[7] R. L. Taylor, P. J. Bereshford and E.L. Wilson, A non-conforming element for stress analysis, International Journal of Numerical Methods in Engineering, 10, 1211-1219, 1976. https://doi.org/10.1002/nme.1620100602

[8] R. D. Cook, Ways to improve the bending response of finite elements, International Journal of Numerical Methods in Engineering, 11, 1029-1039, 1977. https://doi.org/10.1002/nme.1620110609

[9] T. Belytschko, W. K. Liu, B. Moran, Nonlinear Finite Elements for Continua and Structures, Wiley, 2000.

Jouni Freund,

Aalto University, School of Engineering, P.O. Box 12100, FIN-00076 Aalto, Finland jouni.freund@aalto.fi

Eero-Matti Salonen

Sibeliuksenkatu 3 B 25

00250 Helsinki, Finland

eeromatti.salonen@gmail.com 